Editorial

\title{
Acknowledgement to Reviewers of Informatics in 2018
}

Informatics Editorial Office

MDPI, St. Alban-Anlage 66, 4052 Basel, Switzerland

Published: 9 January 2019

Rigorous peer-review is the corner-stone of high-quality academic publishing. The editorial team greatly appreciates the reviewers who contributed their knowledge and expertise to the journal's editorial process over the past 12 months. In 2018, a total of 43 papers were published in the journal, with a median time to first decision of 24 days and a median time to publication of 68 days. The editors would like to express their sincere gratitude to the following reviewers for their cooperation and dedication in 2018:

\begin{tabular}{ll} 
Agugiaro, Giorgio & Church, E. Mitchell \\
Alves, Tiago & Ciobanu, Dragos \\
Andreasen, Esben Sparre & Cohen, Michael \\
Andrews, Christopher & Cotfas, Liviu-Adrian \\
Anton, David & Cowling, Michael \\
Baker, Thar & Craven, Michael P. \\
Bárcena, Elena & Dai, Hong-Jie \\
Basford, Philip James & De, Suparna \\
Belik, Vitaly & De Carvalho, Rogerio Atem \\
Ben-Othmane, Lotfi & De Meo, Pasquale \\
Bianchi, Alessandro & Deufemia, Vincenzo \\
Biljecki, Filip & Díaz-Cacho, Miguel \\
Bonastre, Alberto & Ding, Meng \\
Boyle, Elizabeth & Dubey, Rameshwar \\
Buckley, Christopher & Elahi, Mehdi \\
Burdett, Robert & Evans, Laura \\
Calero Valdez, André & Fabregat, Ramon \\
Cano Garcia, Jose Manuel & Fiorini, Laura \\
Capitman, John & Fischer, Peter M. \\
Castagnos, Sylvain & Flouris, Giorgos \\
Cavallo, Pierpaolo & Fominykh, Mikhail \\
Cavenaghi, Marcos Antônio & Fonseca, José \\
Ceci, Michelangelo & Fortune, Emma \\
Chae, Junjae & Frey, Lewis \\
Chakraborty, Joyram & Fujinami, Kaori \\
Charvátová, Hana & Fullér, Robert \\
Chen, Tongwen & Gallicchio, Claudio \\
Chen, Shyi-Ming & Gamboa, Hugo \\
Chitkushev, Lou & Garau, Chiara \\
Chiu, Po-Sheng & Ge, Mouzhi \\
Choenni, Sunil & Ge, Mouzhi \\
Chou, Pao-Nan & Georgakis, Panos \\
Christen, Markus & Ghanavati, Sepideh \\
& \\
\hline
\end{tabular}


Ghanipoor Machiani, Sahar

Giannelli, Carlo

Gilson, Fabian

Govender, Irene

Gravina, Raffaele

Guan, Yu

Gunawardana, Upul

GÜRCAN, Önder

Gwak, Jeonghwan

Hachaj, Tomasz

Hachour, Samir

Hammond, William

Han, Fei

Han, Jayoung

Hanke, Sten

Harding, Chris

Hegde, Nagaraj

Hek, Karin

Hinkelmann, Knut

Holzinger, Andreas

Hoque, Enamul

Horváth, Tomáš

Hussey, Pamela

Hwang, Ting-Kai

Iglesias Martínez, José Antonio

Ishaq, Isam

Iwan, Stanisław

Jalal, Ahmad

Jayarathna, Sampath

Jiang, Qi

Jiang, Crystal L.

Jin, Ge

Jovanovic, Petar

Kangin, Dmitry

Karamitsos, Ioannis

Katifori, Akrivi

Kelly, Ryan

Khan, Imran

Khorasani, Elham Sahebkar

Kico, Iris

Kim, Jinmo

Kolomvatsos, Kostas

Koohikamali, Mehrdad

Korkontzelos, Yannis

Koskimäki, Heli

Koutromanos, Georgios

Kurasova, Olga

Kwak, Daehan

Läubli, Samuel

Le, Zhang

Lee, Dong Joon

Lee, Hao-Chih
Lehavi, Amnon

Leightley, Daniel

Leung, Carson K.

Liagouris, John

Lim, Jiyoung

Liu, Yu-Chuan

Lo, Yu-Sheng

Luis, Martínez López

Magoulas, George D.

Magoulas, George

Malmström, Lars

Manuri, Federico

Marai, G. Elisabeta

Matheny, Michael

Mattoso, Marta

McCullagh, Paul

Merrill, Jacqueline A.

Miao, Hui

Mikalef, Patrick

Mikropoulos, Tassos A.

Missier, Paolo

Muñoz, Ricardo F.

Murta, Leonardo

Naeem, Usman

Olaru, Andrei

Oneto, Luca

Ovalle, María Antonia

Pan, Cuiping

Panciroli, Chiara

Pankowska, Malgorzata

Papapanagiotou, Petros

Pareschi, Remo

Parisi, German I.

Parsons, Paul

Pasupathy, Kalyan S.

Payeur, Pierre

Perallos Ruiz, Asier

Perez, Alfredo

Pérez-Navarro, Antoni

Perikos, Isidoros

Pieri, Gabriele

Pilkington, Marc

Raghupathi, Wullianallur

Randall, David

Ravì, Daniele

Rawassizadeh, Reza

Ringsberg, Henrik

Romero Brufau, Santiago

Rosaci, Domenico

Roth, Christian

Rula, Anisa

Ruotolo, Francesco 
Sahama, Tony

Sallaberry, Arnaud

Samaras, Achilleas

Samoilenko, Anna

San-Segundo, Rubén

Santoro, Donatello

Sargano, Allah Bux

Schneider, Gerold

Schulze, Jurgen P.

Schwarz, Chris

Sedig, Kamran

Seitz, Jochen

Serrano, Manuel

Shirahama, Kimiaki

Sik Lanyi, Cecilia

Simini, Filippo

Sobrino, Xosé Antón Vila

Spanakis, Emmanouil G.

Stavrou, Eliana

Suarez, Alvaro

Szirbik, Nick B.

Thakur, Subhasis

Tiakas, Eleftherios

Toreini, Ehsan

Torres Hostench, Olga
Triviño, Alicia

Turkay, Cagatay

Van Laerhoven, Kristof

Vatavu, Andrei

Verhoeven, Peter

Vlahu-Gjorgievska, Elena

Volná, Eva

Wang, Chih-Hsuan

Wang, Sheng-Shih

Wang, Chen

Wang, Lipo

Warncke-Wang, Morten

Weller, Steven

Wersing, Heiko

Wilson, Eddie

Wöhrer, Alexander

Woznowsk, Pete

Wu, Shuheng

Wysocki, Marian

$\mathrm{Xu}$, Xinhao

Yang, Geng

Zegarra Flores, Jesus

Zhao, Xiang

Ziegler, Jürgen

Zohuri, Bahman

(C) 2019 by the author. Licensee MDPI, Basel, Switzerland. This article is an open access article distributed under the terms and conditions of the Creative Commons Attribution (CC BY) license (http://creativecommons.org/licenses/by/4.0/). 